\title{
Cryogenic Magnetocaloric Effect in a Ferromagnetic Molecular Dimer
}

\author{
Marco Evangelisti,* Olivier Roubeau, Elias Palacios, Agustín Camón, Thomas N. Hooper, Euan K. \\ Brechin, and Juan J. Alonso
}

Over the last few years, great interest has emerged in the synthesis and magnetothermal studies of polymetallic molecular clusters based on paramagnetic ions, often referred to as molecular nanomagnets, in view of their potential application as lowtemperature magnetic refrigerants. ${ }^{[1,2]}$ What makes them promising is that their cryogenic magnetocaloric effect (MCE) can be considerably larger than that of any other magnetic refrigerant, e.g. lanthanide alloys and magnetic nanoparticles. ${ }^{[3]}$ The MCE is the change of magnetic entropy $\left(\Delta S_{m}\right)$ and related adiabatic temperature $\left(\Delta T_{a d}\right)$ following the change of applied magnetic field and it can be exploited for cooling applications via a field removal process called adiabatic demagnetization. Although the MCE is intrinsic to any magnetic material, in only a few cases are the changes sufficiently large to make them suitable for applications. The ideal molecular refrigerant comprises the following key characteristics: ${ }^{[1]}$ (i) a large spin ground state $S$, since the magnetic entropy amounts to $R \ln (2 S+1)$; (ii) a negligible magnetic anisotropy, which permits easy polarization of the net molecular spins in magnetic fields of weak or moderate strength; (iii) the presence of low-lying excited spin states, which enhances the field dependence of the MCE due to the increased number of populated spin states; (iv) dominant ferromagnetic exchange, ${ }^{[3(\mathrm{c})]}$ favouring a large $S$ and hence a large field dependence of the MCE; $(v)$ a relatively low molecular mass (or a large metal:ligand mass ratio) since the non-magnetic ligands contribute passively to the MCE. Although this last point is crucial for obtaining an enhanced effect, it has been mostly ignored to date. Molecular cluster compounds tend to have a very low magnetic density because of the large complex structural frameworks required to encase the multi-metallic core.

In this communication we propose a drastically different

[*] Dr. M. Evangelisti, Dr. O. Roubeau, Prof. E. Palacios, Dr. A. Camón,

Instituto de Ciencia de Materiales de Aragón (ICMA)

CSIC-Universidad de Zaragoza, Departamento de Física de la Materia Condensada, 50009 Zaragoza (Spain)

Fax: (+34) 976761229

E-mail: evange@unizar.es

Homepage: http://molchip.unizar.es/

T. N. Hooper, Dr. E. K. Brechin

EaStCHEM School of Chemistry

The University of Edinburgh

West Mains Road, Edinburgh, EH9 3JJ, Scotland (UK)

Prof. J. J. Alonso

Departamento de Física Aplicada I

Universidad de Málaga, 29071 Málaga (Spain)

[**] This work was supported by the Spanish MICINN through grants MAT2009-13977-C03, MAT2007-61621 and CSD2007-00010. EKB thanks the EPSRC. approach by focusing on the simple and well-known ferromagnetic molecular dimer gadolinium acetate tetrahydrate, ${ }^{[4]}$ $\left[\left\{\mathrm{Gd}(\mathrm{OAc})_{3}\left(\mathrm{H}_{2} \mathrm{O}\right)_{2}\right\}_{2}\right] \cdot 4 \mathrm{H}_{2} \mathrm{O}(\mathbf{1})$. The structure of $\mathbf{1}$ is depicted in Figure 1 and comprises a dimer of $\mathrm{Gd}^{3+}$ ions bridged through two of the six carboxylate groups which bond in a $\eta^{2}: \eta^{1}: \mu_{2}$-fashion. The remaining acetates are chelating with the nine-coordinate [capped square anti-prismatic] geometry of the metal centres being completed by the presence of two terminally bound $\mathrm{H}_{2} \mathrm{O}$ molecules. These partake in intra-molecular H-bonding to the neighbouring chelating acetate ligands, and are responsible for both the direct inter-molecular $\mathrm{H}$-bonds in the $a-b$ plane and the inter-plane $\mathrm{H}$ bonds mediated by the lattice $\mathrm{H}_{2} \mathrm{O}$ molecules (Fig. S1 and Table S1).

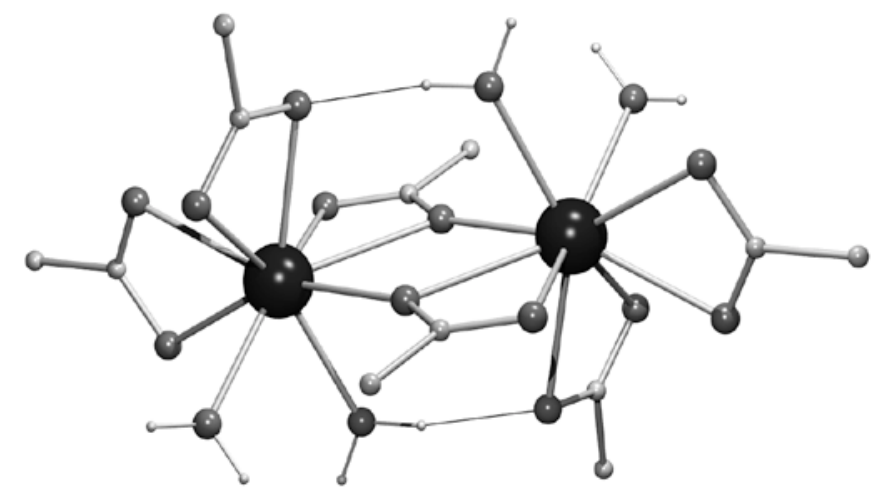

Figure 1. The molecular structure of 1 . Code: $\mathrm{Gd}=$ black, $\mathrm{O}=$ dark grey, $\mathrm{C}$ = light grey, $\mathrm{H}=$ small bullets. $\mathrm{H}$-atoms of the methyl groups are omitted for clarity. Intramolecular hydrogen bonds are depicted as thin lines.

Our theoretical and experimental investigations (see Supporting Information for details) of the magnetothermal properties of $\mathbf{1}$ down to millikelvin temperatures reveal a truly enormous MCE. In addition to magnetization and heat capacity experiments, which we employ to indirectly estimate the MCE, we make use of a homemade experimental set-up that allows us to measure both $\Delta S_{m}$ and $\Delta T_{a d}$, ${ }^{[5]}$ thus directly probing the extraordinary cooling performance of $\mathbf{1}$.

Figure 2 depicts the dc magnetic susceptibility $(\chi)$ of $\mathbf{1}$ collected in an applied field $B_{0}=0.1 \mathrm{~T}$ over the $2-300 \mathrm{~K}$ temperature range. The room-temperature experimental value of $\chi T$ agrees with that expected from two non-interacting $\mathrm{Gd}^{3+}$ ions with $g=2$, i.e. $\chi T=$ $15.75 \mathrm{~cm}^{3} \mathrm{~K} \mathrm{~mol}^{-1}$. On lowering $T, \chi T$ stays nearly constant with decreasing temperature down to $\sim 20 \mathrm{~K}$, below which it increases significantly reaching a value of $18.8 \mathrm{~cm}^{3} \mathrm{~K} \mathrm{~mol}^{-1}$ at $2 \mathrm{~K}$, corroborating the dominant ferromagnetic coupling between the two $\mathrm{Gd}^{3+}$ ions within each molecular unit. The isothermal molar magnetization $\left(M_{m}\right)$ measurements (inset of Fig. 2) shows a saturation value of $14.0 N \mu_{B}$ at the lowest investigated $T$ of $2 \mathrm{~K}$, in agreement with the ferromagnetic spin state $S=7$. In order to estimate the intramolecular exchange constant, we fitted the experimental $\chi T$ vs. $T$ curve (Fig. 2) to a model based on the isotropic spin Hamiltonian $H=-J\left(S_{\mathrm{Gd} 1} \cdot S_{\mathrm{Gd} 2}\right)$, obtaining $J / k_{B}=$ $0.068(2) \mathrm{K}$ and $g=2.01(1)$, in agreement with previous studies. ${ }^{[4(\mathrm{~b})]}$ 


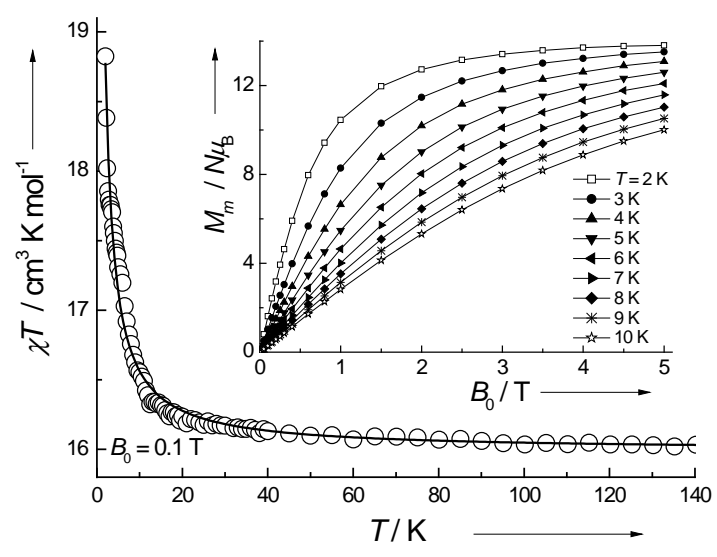

Figure 2. Temperature dependence ( $T>2 \mathrm{~K}$ ) of the dc susceptibility $\chi T$ for $\mathbf{1}$ collected in an applied field of $0.1 \mathrm{~T}$. Line is the fit, see text. Inset: magnetization of $\mathbf{1}$ versus applied field for several temperatures. Lines are guides to the eye.

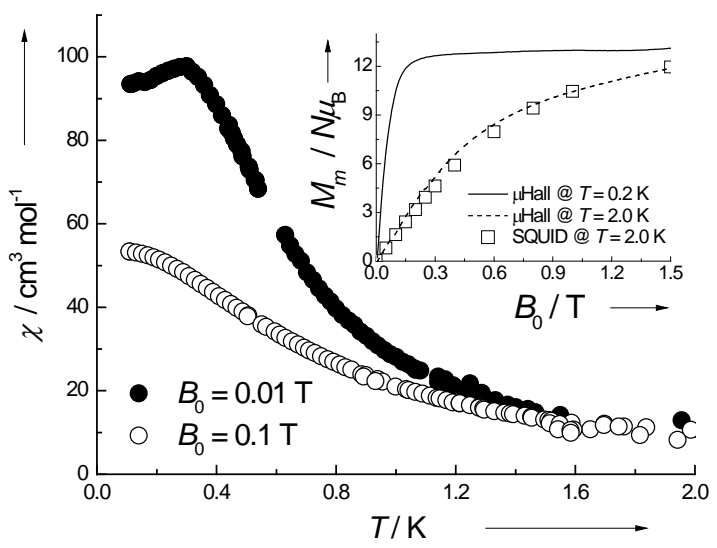

Figure 3. Low-temperature $(T<0.2 \mathrm{~K})$ micro-Hall magnetometry for $\mathbf{1}$. Temperature dependence of the dc-susceptibility $\chi$ collected in applied fields of 0.01 and $0.1 \mathrm{~T}$. Inset: experimental molar magnetization versus applied field for the indicated temperatures.

By Hall-based $\mu$-magnetometry, we extended the magnetic measurements down to lower temperatures (Fig. 3), with the isothermal $M_{m}\left(B_{0}\right)$ curve collected for $T=0.2 \mathrm{~K}$ (inset) supporting the dominant ferromagnetism of $\mathbf{1}$. The $T$-dependence of the dc susceptibility deviates from the Curie law at the lowest temperatures, giving rise to an anomaly centred at $\approx 0.3 \mathrm{~K}$ for $B_{0}=0.01 \mathrm{~T}$. The slight decrease in $\chi$ below this temperature and the disappearance of the anomaly for fields higher than $0.1 \mathrm{~T}$ (Fig. 3) suggest that the complex undergoes a transition to a magnetically ordered phase in which ferromagnetic interactions are important. In order to further elucidate the mechanism of magnetic ordering, we have performed numerical simulations following the standard Metropolis Monte Carlo algorithm. ${ }^{[6]}$ We have considered each $\left[\mathrm{Gd}_{2}\right]$ complex as an isotropic point dipole, i.e. we assume a ferromagnetic $J=\infty$ intramolecular $\mathrm{Gd}^{3+} \cdots \mathrm{Gd}^{3+}$ exchange coupling and no intermolecular exchange paths. In satisfactory agreement with the experimental observations, we obtain a critical temperature of $\approx 0.18 \mathrm{~K}$ for $B_{0}=0$ and a magnetic structure formed by alternating ferromagnetic $a-b$ planes (see Supporting Information). We next turn to the evaluation of the magnetothermal properties of $\mathbf{1}$ by presenting its experimental heat capacity $(C)$. Figure 4 (top) depicts the experimental $T$-dependence of $C$ for selected applied fields. At high temperatures the heat capacity is dominated by non-magnetic contributions arising from thermal vibrations of the lattice, which can be modelled with the six-fold Debye function (dashed line in Fig. 4) yielding a value of $\Theta_{D}=61.6$ $\mathrm{K}$ for the Debye temperature, typical for this class of cluster compound. $^{[7]}$ At low temperatures the applied field splits the molecular spin multiplet $S=7$, gradually decoupling the intramolecular $\mathrm{Gd}^{3+} \cdots \mathrm{Gd}^{3+}$ exchange coupling and giving rise to a broad (Schottky-type) feature, which shifts to higher $T$ by increasing $B_{0}$. Unfortunately, the experimentally accessible temperatures do not permit the observation of the phase transition. We note however that the zero-field $C$ keeps increasing by decreasing $T$ in the lowest temperature region, reaching values exceeding that expected for magnetically isolated $\left[\mathrm{Gd}_{2}\right]$ units $(\cong 1.9 R)$. From the experimental heat capacity, the temperature dependence of the magnetic entropy $\left(S_{m}\right)$ is obtained by integration, using $S_{m}(T)=\int C_{m} / T \mathrm{~d} T$, where the magnetic heat capacity $C_{m}$ is obtained from $C$ upon subtracting the lattice contribution. The so-obtained $S_{m}(T)$ curves are shown in the bottom panel of Figure 4 for the corresponding applied fields. As expected, $S_{m}$ for $B_{0}>0$ tends to the maximum entropy value per mole involved at high temperatures, corresponding to two $\mathrm{Gd}^{3+} s=$ $7 / 2$ spins $(2 \cdot R \ln (2 s+1) \cong 4.16 R)$. In the case of $B_{0}=0$, our experimental blindness for $T$ lower than $\approx 0.35 \mathrm{~K}$ forced us to add a constant value to the zero-field $S_{m}(T)$ in order to match the limiting value at high $T$. As we shall see below, this crude procedure does not jeopardize our evaluation of the MCE of $\mathbf{1}$.

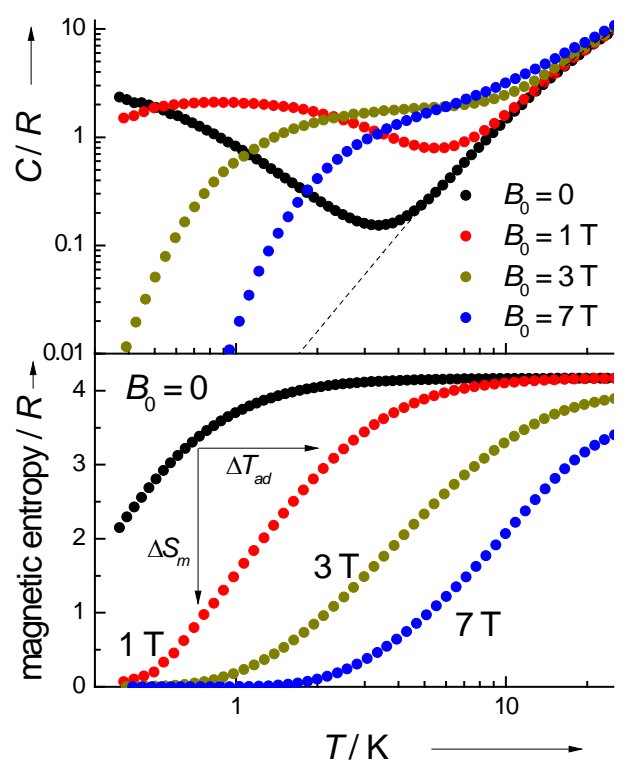

Figure 4. Top: temperature dependencies of the heat capacity of $\mathbf{1}$ normalized to the gas constant $R$ collected for $B_{0}=0,1,3$ and 7 T. Bottom: temperature dependencies of the experimental magnetic entropy for several $B_{0}$, as obtained from the respective heat capacity data after subtracting the lattice contribution (dashed line).

From the $S_{m}$ data, it is then straightforward to obtain the changes of magnetic entropy and adiabatic temperature, both indicated by arrows in Fig. 4. The results are shown in Figure 5 as a function of $T$ and for several field changes $\Delta B_{0}=B_{f}-B_{i}$. A striking result is the $-\Delta S_{m}$ which can be seen to reach values over $40 \mathrm{~J} \mathrm{~kg}^{-1} \mathrm{~K}^{-1}$ at $T \approx 1.8$ $\mathrm{K}$ for $\Delta B_{0}=7 \mathrm{~T}$, much larger than any other value reported in the recent literature. ${ }^{[3]}$ We note that $-\Delta S_{m}$ approaches the maximum entropy value per mole, $4.16 R \cong 42.5 \mathrm{~J} \mathrm{~kg}^{-1} \mathrm{~K}^{-1}$, for two fully decoupled $\mathrm{Gd}^{3+}$ ions. If $\Delta B_{0}$ is lower, or of the order of, the strength of the ferromagnetic intramolecular $\mathrm{Gd}^{3+} \cdots \mathrm{Gd}^{3+}$ exchange 
coupling, then the low-temperature $-\Delta S_{m}$ is much larger than can be produced in the absence of such a coupling. For instance, a relatively modest $\Delta B_{0}=1 \mathrm{~T}$ is already sufficient to provide a $-\Delta S_{m}$ as large as $27 \mathrm{~J} \mathrm{~kg}^{-1} \mathrm{~K}^{-1}$ at $T \approx 0.5 \mathrm{~K}$. This remarkable field dependence of the MCE is also observed in $\Delta T_{a d}$. Figure 5 (bottom) shows that $\Delta B_{0}=7 \mathrm{~T}$ provides a maximum $\Delta T_{a d}=12.7 \mathrm{~K}$ for the same temperatures in which we observe the $-\Delta S_{m}$ maxima. By lowering $\Delta B_{0}$ to 3 and $1 \mathrm{~T}, \Delta T_{a d}$ decreases to 9.0 and $3.5 \mathrm{~K}$, respectively. Therefore, the field dependence of $\Delta T_{a d}$ increases from nearly 2 to well over $3 \mathrm{~K} / \mathrm{T}$, respectively, setting this material as the most efficient refrigerant for this low- $T$ region. ${ }^{[8]}$

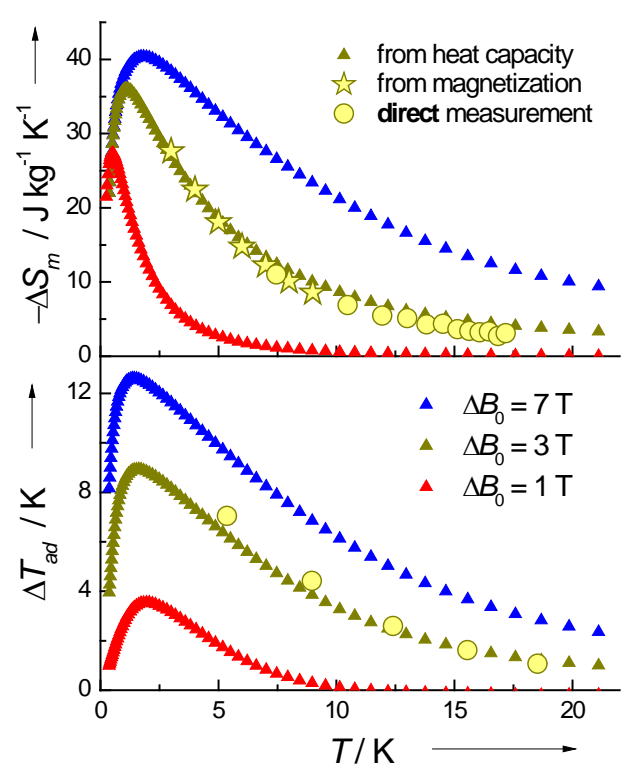

Figure 5. Top: temperature dependencies of the magnetic entropy change $\Delta S_{m}(T)$ as indirectly obtained from heat capacity (triangles) and magnetization (stars) data, together with the direct measurements (dots), for the indicated applied-field changes $\Delta B_{0}$. Bottom: temperature dependencies of the adiabatic temperature change $\Delta T_{a d}(T)$ as indirectly obtained from heat capacity data, together with the direct measurements, for the indicated $\Delta B_{0}$.

In addition to heat capacity, magnetization data can also be employed for estimating $\Delta S_{m}$ by making use of the Maxwell relation, $\Delta S_{m}(T)=\int\left[\partial M_{m}\left(T, B_{0}\right) / \partial T\right] \mathrm{d} B_{0}$. From the isothermal $M_{m}\left(B_{0}\right)$ curves of Fig. 2, the so-obtained $\Delta S_{m}(T)$ for $\Delta B_{0}=3 \mathrm{~T}$ is displayed in Fig. 5 (top) and can be seen to be in perfect agreement with the data obtained from $C$, proving that our experimental uncertainty in the zero-field $S_{m}(T)$ does not affect the evaluation of $\Delta S_{m}$ and $\Delta T_{a d}$ of $\mathbf{1}$. However, we note that both indirect procedures we followed for obtaining the MCE rely on numerical integrations that, by their nature, can be the source of large errors. ${ }^{[9]}$ To overcome any possible shortfall inherent to these approaches, we have also measured $\Delta S_{m}$ and $\Delta T_{a d}$ directly under quasi-adiabatic conditions (see Fig S3). ${ }^{[5]}$ By again considering $\Delta B_{0}=3 \mathrm{~T}$, we obtain the $\Delta S_{m}$ and $\Delta T_{a d}$ values depicted in Fig. 5 , which rather beautifully corroborate our previous estimates.

In conclusion, an unprecedentedly large magnetocaloric effect at extremely low $T$ is reported to occur in the simple ferromagnetic molecular dimer, gadolinium acetate tetrahydrate. The magnetic ordering originates from dipolar coupling, and its collective magnetic behaviour is somewhat reminiscent of that reported for gadolinium sulphate octahydrate, a well-studied, purely dipolar system, which was the subject of the very first adiabatic demagnetization experiments. ${ }^{[10]}$ The enormous advantage of $\mathbf{1}$ over this prototype magnetic coolant is the intramolecular ferromagnetic exchange coupling, which favours the field-dependent enhancement of the magnetocaloric effect.

\section{Experimental Section}

See Supporting Information for a description of the experimental methods; structure of 1 , collected at $T=100 \mathrm{~K}$ in CIF format, CCDC $\mathrm{n}^{\circ} 820956$, and views of the hydrogen bonding networks in 1; Monte Carlo numerical simulations of the magnetic ordering; representative direct measurement of the temperature evolution of 1 under quasiadiabatic conditions.

Received: ((will be filled in by the editorial staff))

Published online on ((will be filled in by the editorial staff))

Keywords: gadolinium · low-temperature physics $\cdot$ magnetic properties $\cdot$ magnetocaloric effect $\cdot$ molecular refrigerant

[1] For a recent review, see M. Evangelisti, E. K. Brechin, Dalton Trans. 2010, 39, 4672, and references therein.

[2] a) Yu. I. Spichkin, A. K. Zvezdin, S. P. Gubin, A. S. Mischenko, A. M. Tishin, J. Phys. D: Appl. Phys. 2001, 34, 1162; b) F. Torres, J. M. Hernández, X. Bohigas, J. Tejada, Appl. Phys. Lett. 2000, 77, 3248; c) F. Torres, X. Bohigas, J. M. Hernández, J. Tejada, J. Phys.: Condens. Matter 2003, 15, L119; d) M. Affronte, A. Ghirri, S. Carretta, G. Amoretti, S. Piligkos, G. A. Timco, R. E. P. Winpenny, Appl. Phys. Lett. 2004, 84, 3468; e) J. Schnack, R. Schmidt, J. Richter, Phys. Rev. B 2007, 76, 054413.

[3] a) M. Evangelisti, A. Candini, A. Ghirri, M. Affronte, E. K. Brechin, E. J. L. McInnes, Appl. Phys. Lett. 2005, 87, 072504; b) R. Shaw, R. H. Laye, L. F. Jones, D. M. Low, C. Talbot-Eeckelaers, Q. Wei, C. J. Milios, S. J. Teat, M. Helliwell, J. Raftery, M. Evangelisti, M. Affronte, D. Collison, E. K. Brechin, E. J. L. McInnes, Inorg. Chem. 2007, 46, 4968; c) M. Manoli, R. D. L. Johnstone, S. Parsons, M. Murrie, M. Affronte, M. Evangelisti, E. K. Brechin, Angew. Chem. 2007, 119, 4540; Angew. Chem., Int. Ed. 2007, 46, 4456; d) M. Manoli, A. Collins, S. Parsons, A. Candini, M. Evangelisti, E. K. Brechin, J. Am. Chem. Soc. 2008, 130, 11129; e) M. Evangelisti, A. Candini, M. Affronte, E. Pasca, L. J. de Jongh, R. T. W. Scott, E. K. Brechin, Phys. Rev. B 2009, 79, 104414; f) G. Karotsis, M. Evangelisti, S. J. Dalgarno, E. K. Brechin, Angew. Chem. 2009, 121, 10112; Angew. Chem., Int. Ed. 2009, 48, 9928; g) G. Karotsis, S. Kennedy, S. J. Teat, C. M. Beavers, D. A. Fowler, J. J. Morales, M. Evangelisti, S. J. Dalgarno, E. K. Brechin, J. Am. Chem. Soc. 2010, 132, 12983; h) S. Nayak, M. Evangelisti, A. K. Powell, J. Reedijk, Chem. Eur. J. 2010, 16, 12865; i) Y.-Z. Zheng, M. Evangelisti, R. E. P. Winpenny, Chem. Sci. 2011, 2, 99; j) Y.-Z. Zheng, M. Evangelisti, R. E. P. Winpenny, Angew. Chem. 2011, 123, 3776; Angew. Chem., Int. Ed. 2011, 50, 3692; l) S. K. Langley, N. F. Chilton, B. Moubaraki, T. Hooper, E. K. Brechin, M. Evangelisti, K. S. Murray, Chem. Sci. 2011, DOI: $10.1039 / \mathrm{c} 1 \mathrm{sc} 00038$.

[4] a) M. C. Favas, D. L. Kepert, B. W. Skelton, A. H. White, J. Chem. Soc. Dalton Trans. 1980, 454; b) S. T. Hatscher, W. Urland, Angew. Chem. 2003, 115, 2969; Angew. Chem., Int. Ed. 2003, 42, 2862.

[5] a) L. Tocado, Ph.D. Thesis, Universidad de Zaragoza, 2008; b) L. Tocado, E. Palacios, R. Burriel, J. Magn. Magn. Mater. 2005, 290291, 719 .

[6] N. A. Metropolis, A. W. Rosenbluth, M. N. Rosenbluth, A. H. Teller, E. Teller, J. Chem. Phys. 1953, 21, 1087.

[7] M. Evangelisti, F. Luis, L. J. de Jongh, M. Affronte, J. Mater. Chem. 2006, 16, 2534.

[8] For an overview see, e.g., V. K. Pecharsky, K. A. Gschneidner Jr., J. Magn. Magn. Mater. 1999, 200, 44, and references therein.

[9] V. K. Pecharsky, K. A. Gschneidner, J. Appl. Phys. 1999, 86, 565.

[10] a) W. F. Giauque, D. P. MacDougall, Phys. Rev. B 1935, 43, 768; b) W. F. Giauque, D. P. MacDougall, J. Am. Chem. Soc. 1935, 57, 1175; c) R. F. Wielinga, J. Lubbers, W. J. Huiskamp, Physica 1967, 37, 375. 
